\title{
Investigation of the antioxidant capacity of the components of Astragalus after membrane separation
}

\author{
Yandong Wang ${ }^{1}$, Yafei Guo ${ }^{2}$, Yingli Wang ${ }^{2}$ and Zemin Zhang ${ }^{1 *}$ \\ ${ }^{1}$ State Key Laboratory for Conservation and Utilization of Subtropical Agro-Bioresources, Guangdong Provincial Key Laboratory of \\ Plant Molecular Breeding, South China Agricultural University, Guangzhou 510642, China \\ ${ }^{2}$ Institute of Pharmaceutical \& Food Engineering, Shanxi University of Chinese Medicine, 030619 Jinzhong, Shanxi, China
}

\begin{abstract}
Astragalus is commonly used in health supplements, and its flavonoids and saponins are the important material basis for immune system enhancement. The study on the effective component contents and antioxidant capacities of astragalus extract after membrane separation lays the foundation of the application of membrane separation technology in health supplement development. The astragalus extract was filtered by suction and passed through membranes of $10000 \mathrm{Da}, 2500 \mathrm{Da}$, and $600 \mathrm{Da}$ to obtain retentate 1 (M1), retentate 2 (M2), retentate 3 (M3) and permeate MT. UV/vis spectrophotometer was used to compare the contents of total flavonoids and total saponins before and after each step of membrane separation. High performance liquid chromatography (HPLC) was used to analyze the contents of Verbasil Glucoside and Astragaloside IV of all membrane separation samples, and the antioxidant activities were determined. The contents of flavonoids in membrane separation samples were significantly different. In the freeze-dried powders obtained from the membrane separation, the contents of total flavonoids and Verbasil Glucoside were the lowest in MT, M1 was the highest, and M3 was the second highest. The order of contents of total saponins and Astragaloside IV of the freeze-dried powders from membrane separation was as follows: M3 $>$ M1 $>$ M2 $>$ MT. Among them, the content of total saponins in the freeze-dried powder of M3 was the highest, which reached 2.67 times of that of the astragalus extract. The order of the scavenging activities of membrane separation samples for DPPH free radical was: MT $>$ M3 $>$ M2 $>$ M1 $>$ astragalus extract. The strongest scavenging activity of hydroxyl free radicals was found in M3, and the scavenging rates of hydroxyl free radicals in all samples were higher than those of VC. The total antioxidant capacity of FRAP showed a certain dose-effect relationship. At the same concentration, the FRAP values of M1 and MT were higher than other samples. Membrane separation technology can be used to separate and purify the effective components from astragalus extract. M3 has the highest contents of the total flavonoids and total saponins, and its antioxidant capacity is better than that of astragalus extract and other samples obtained by membrane separation.
\end{abstract}

\section{Introduction}

Astragalus is a common ingredient used to produce health supplements, and it has been broadly planted globally [1, 2]. The most widely used astragalus are Astragalus membranaceus (Fisch.) Bge. var. mongholicus (Bge.) Hsiao and Astragalus membranaceus (Fisch.) Bge ${ }^{[3]}$. The components, including flavonoids and saponin-like ingredient, in astragalus play an important role as the material basis for immune system enhancement ${ }^{[4-6]}$. Macro-porous resin chromatography has been applied in the separation and purification of flavonoids and saponin-like ingredient of astragalus. However, the introduction of organic reagents during the purification process and low production efficiency are two major issues ${ }^{[7]}$. To reduce the residue of the organic reagents, the study of membrane separation technique for the purification of active ingredients of astragalus is of practical significance ${ }^{[8-11]}$.
In our project, astragalus extract will be separated and purified using membrane separation. The amount of active ingredients and their antioxidant capacities after purification by different membrane separation will be examined. This project is going to provide the basis for the application of membrane separation technique in the health product area.

\section{Materials and Equipment}

\subsection{Materials and reagents}

Astragalus membranaceus (Fisch.) Bge. Var. mongholicus (Bge.) Hsiao, Calycosin-7-O- $\beta$-D-glucoside (batch number: 111920-201606, Chinese National Institutes for Food and Drug Control), Astragaloside IV (batch number: 110781-201616, Chinese National Institutes for Food and Drug Control), Methanol (HPLC

\footnotetext{
* Corresponding author: E-mail: zmzhang@scau.edu.cn, Tel: 020-85280800
} 
grade, batch number: 20171201, Damao Chemical Reagent Factory), Acetonitrile (HPLC grade, batch number: 20120413, Tianjin Kemiou Chemical Reagent Co., Ltd.), Formic acid (HPLC grade, batch number: 20161011, Damao Chemical Reagent Factory), Aluminum Nitrate (batch number: 20160808, Tianjin Tianli Chemistry Reagent Co., Ltd.), Sodium Nitrite (batch number: 20160818, Tianjin Tianli Chemistry Reagent Co., Ltd.), Sodium Hydroxide (batch number: 20110104, Tianjin University Chemical Industry), Absolute Ethanol (batch number: 20160901, Tianjin Beichenfangzheng Chemical Reagent Factory), Vanillin and Phenol (Damao Chemical Reagent Factory), 1,1diphenyl-2-picrylhydrazyl (DPPH, TCI), 3-(2-Pyridyl)5,6-diphenyl-1,2,4-triazine (TPTZ, Nanjing Duly Biotech Co., Ltd), Vitamin C (Vc, Shanxi Taiyuan Pharmaceutical Industry Co., Ltd.), $\mathrm{H}_{2} \mathrm{O}_{2}$ (batch number: 20160607), Acetic Acid (batch number: 20090210), Ferrous Sulfate (batch number: 20160223), Sodium Acetate (batch number: 20130828), $\mathrm{FeCl}_{3}$ (batch number: 20100910), Phenanthroline, ferrous sulfate, sodium dihydrogen phosphate, sodium hydrogen phosphate (Tianjin Kemiou Chemical Reagent Co., Ltd.).

\subsection{Equipment}

LNG-NF-101 ultrafiltration membrane separation apparatus (laboratory scale) with $10 \mathrm{kDa}$ molecular weight cut-off (MWCO) polyethersulfone ultrafiltration membrane (No. S-UF10, S113310397), 2.5 kDa MWCO polyamide ultrafiltration membrane (No. S-UF2.5, S15310107), and 600 Da MWCO polyamide ultrafiltration membrane (No. S-NF600, S12910174) (Shanghai Laungy membrane separation, China).

ACQUITY Ultra Performance Liquid Chromatography (Waters (Shanghai), USA), UntiMate 3000 High Performance Liquid Chromatography (Thermo Sicentific, USA), TU-1810 UV/vis spectrometer (Beijing Persee, China), RE-52AA Rotary Evaporator (Shanghai Yarong Biochemistry Instrument, China), CP124 C Scale (Ohaus Instrument (Shanghai), USA), Christ Alpha 1-4 LD Plus Freeze Dryer (BMH, China), DW-86L728 Freezer (Haier Medical, China).

\section{Methods}

\subsection{Separation and Purification}

$210 \mathrm{~g}$ astragalus was mixed with 10 times water (w/v) and soaked for $30 \mathrm{~min}$. All samples were extracted twice and then combined. Combined sample was cooled till room temperature for later use. $110 \mathrm{~mL}$ filtered sample was taken and topped up with water to $2000 \mathrm{~mL}$. In this project, multi-step membrane separation was used for the purification, as shown in Figure 1, $2000 \mathrm{~mL}$ samples were loaded to the membrane in each step. In the process, polyethersulfone membrane (10 kDa MWCO) was used for the first step. Polyamide membrane (2.5 kDa MWCO) and polyamide membrane $(0.6 \mathrm{kDa} \mathrm{MWCO})$ were used in step 2 and 3 for the separation and purification of active components. After separation, retentate samples
M1, M2, M3 and permeate sample MT were lyophilized for later use.

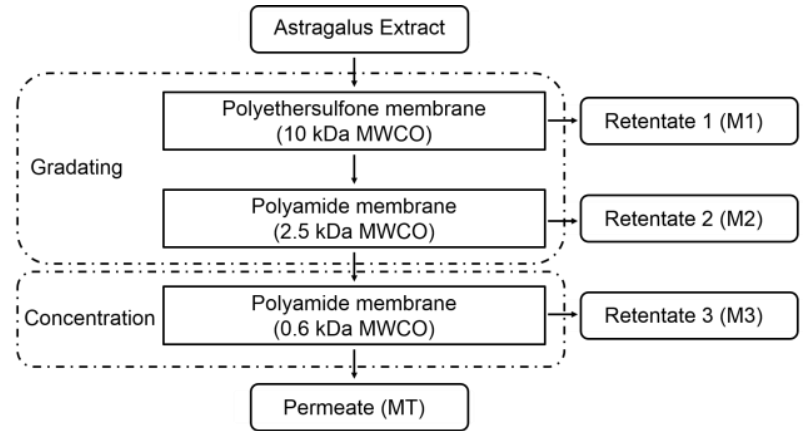

Fig. 1. Flow chart of membrane separation

\subsection{UV-vis spectrophotometry for the measurement of flavonoids and saponins}

Lyophilized powder of M1, M2, M3 and MT samples were dissolved with distilled water. $1.1 \mathrm{~mL}$ of dissolved sample was put into the test tube, and then $0.5 \mathrm{~mL}$ of $5 \%$ Sodium Nitrite was added. The sample was mixed thoroughly and rested for $6 \mathrm{~min}$. After that, $0.5 \mathrm{~mL}$ of $10 \%$ Sodium Nitrite was added. The mixture was mixed and rested for another 6 min. $4 \mathrm{~mL}$ of $1 \mathrm{M} \mathrm{NaOH}$ was added and then the sample was diluted with $70 \%$ Ethanol to $10 \mathrm{~mL}$. Finally, the sample was mixed thoroughly and rested for $15 \mathrm{~min}$. The absorbance at 402 $\mathrm{nm}$ of each sample was measured. The distilled water was used as blank, and Rutin was used for the standard curve. The absorbance was plotted against the concentration. The standard curve is

$$
y=18.3680 x-0.0033 \quad R^{2}=0.9994
$$

$0.1 \mathrm{~mL}$ dissolved M1, M2, M3 and MT samples were mixed with $0.4 \mathrm{~mL}$ absolute ethanol, $0.5 \mathrm{~mL}$ of $8 \%$ Vanillin in absolute ethanol and $5 \mathrm{~mL} 72 \% \mathrm{H}_{2} \mathrm{SO}_{4}$ in the test tubes thoroughly, and immediately placed in the water bath at $62{ }^{\circ} \mathrm{C}$ for $20 \mathrm{~min}$. Then the mixtures were placed in the ice bath to cool to room temperature. The absorbance at $475 \mathrm{~nm}$ of each mixture was measured. The distilled water was used as blank, and Astragaloside IV was used for the standard curve. The absorbance was plotted against the concentration. The standard curve is,

$$
y=1.6275 x-0.0245 \quad \mathrm{R}^{2}=0.9966
$$

\subsection{High-Performance Liquid Chromatography for the measurement of calycosin-7-O- $\beta-D-$ glucoside and Astragaloside IV}

\subsubsection{Measurement of Calycosin-7-O- $\beta$-D-glucoside}

Chromatography column used was Venusil XBP C18 (A) HPLC column $(4.6 \mathrm{~mm} \times 250 \mathrm{~mm}, 5 \mu \mathrm{m})$. Mobile phases were A: $0.2 \%$ Formic acid and B: Acetonitrile. Linear gradient elution: 0-20 min (B: 20\% - 40\%), 20 $30 \mathrm{~min}$ (B: $40 \%$ ). Column temperature was set at $30{ }^{\circ} \mathrm{C}$. $260 \mathrm{~nm}$ absorbance was recorded throughout the whole experiment. Sample loading amount was $10 \mu \mathrm{L}$. 
The peak area of calycosin-7-O- $\beta$-D-glucoside in HPLC was plotted against its concentration and perform the linear regression. The standard curve is:

$$
y=2147.1 x+138032 \quad \mathrm{R}^{2}=0.9900
$$

Lyophilized powder of separated samples was taken and extracted using ultrasonicator in methanol. Solvent was evaporated after filtered with $0.22 \mu \mathrm{m}$ filter membrane, and then methanol was used to dissolve the residue and sample was transferred to the $5 \mathrm{~mL}$ volumetric flask. Samples were then loaded to HPLC and the peak area of each sample was recorded. The peak area was applied to the standard curve to determine the amounts of calycosin-7-O- $\beta$-D-glucoside in M1, M2, M3 and MT samples.

\subsubsection{Measurement of Astragaloside IV}

Chromatography column used for measurement of Astragaloside IV was Venusil XBP C18 (A) HPLC column $(4.6 \mathrm{~mm} \times 250 \mathrm{~mm}, 5 \mu \mathrm{m})$. Mobile phase was $32 \%$ Acetonitrile (A) and $68 \%$ water (B) mixture. Flow rate was set at $1.0 \mathrm{~mL} / \mathrm{min}$. Column temperature was set at $30{ }^{\circ} \mathrm{C}$. Absorbance at $205 \mathrm{~nm}$ was recorded. $20 \mu \mathrm{L}$ sample was loaded.

Lyophilized powder of separated samples was taken and dissolved in $10 \mathrm{~mL}$ distilled water, respectively. Astragaloside IV standard sample was taken and dissolved in methanol at the concentration of 0.51 $\mathrm{mg} / \mathrm{mL}$ as the standard solution. Two-point method in HPLC was used for the measurement of the amount of Astragaloside IV in each sample.

\subsection{Antioxidant capacity assay}

\subsubsection{Measurement of free radical's clearance ability of DPPH}

M1, M2, M3, MT and VC samples with different concentrations including $2 \mathrm{mg} / \mathrm{mL}, 4 \mathrm{mg} / \mathrm{mL} 6 \mathrm{mg} / \mathrm{mL}, 8$ $\mathrm{mg} / \mathrm{mL}$ and $10 \mathrm{mg} / \mathrm{mL}$ were prepared. $0.5 \mathrm{~mL}$ of prepared sample was mixed with $2.5 \mathrm{~mL}$ of $0.084 \mathrm{mg} / \mathrm{L}$ DPPH. $0.5 \mathrm{~mL}$ of distilled water was mixed with $2.5 \mathrm{~mL}$ DPPH as the control. After the mixture was reacted in dark for $30 \mathrm{~min}$, the absorbance at $517 \mathrm{~nm}(A s)$ of each sample was measured using UV-vis spectrophotometer. The absorbance at $517 \mathrm{~nm}$ of absolute ethanol $(A c)$ was used as the blank for the measurement ${ }^{[12]}$. The free radical clearance rate of DPPH was calculated using Equation 1 with the average of three repeated parallel experiments,

Clearance rate $(\%)=(1-A s / A c) \times 100 \%$

\subsubsection{Clearance ability for free hydroxyl radical}

$1 \mathrm{~mL}$ of previous solution in section 3.4.1 of M1, M2, M3, MT and VC samples was taken. $1 \mathrm{~mL}$ of $0.75 \mathrm{mM} \mathrm{1,}$ 10-phenanthroline and $2 \mathrm{~mL}$ of $0.15 \mathrm{M}$ PBS were added to the sample and mixed thoroughly. Then $1 \mathrm{~mL}$ of 0.75
$\mathrm{mM}$ ferrous sulfate and $0.1 \% \mathrm{H}_{2} \mathrm{O}_{2}$ were added, respectively. The mixture was reacted in the water bath at $37{ }^{\circ} \mathrm{C}$ for $1 \mathrm{~h}$, and then the absorbance of each sample at $510 \mathrm{~nm}(B s)$ was measured. The distilled water was used as control. In the measurement of absorbance, the same volume of distilled water was measured as $B b$ and the same volume of distilled water and $\mathrm{H}_{2} \mathrm{O}_{2}$ mixture was measured as $B c^{[13]}$. The clearance rate of hydroxyl radical was calculated using Equation 2:

Clearance rate of $\mathrm{OH}(\%)=((B s-B c)) /((B b-B c)) \times 100 \%(2)$

\subsubsection{Ferric reducing antioxidant power (FRAP) assay}

$200 \mu \mathrm{L}$ of M1, M2, M3, MT and VC solution were taken. Samples were mixed with $6 \mathrm{~mL}$ FRAP working solution and $600 \mu \mathrm{L}$ of distilled water. The samples were then placed in the water bath at $37{ }^{\circ} \mathrm{C}$ for reaction for $10 \mathrm{~min}$. The absorbance of each sample at $593 \mathrm{~nm}$ was measured. Different concentrations of $\mathrm{FeSO}_{4}$ were used for the standard curve. The antioxidant capacity of the sample is linear positively related to its absorbance at $513 \mathrm{~nm}$. The values of the antioxidant capacity of the samples were illustrated using FRAP value $\left(1 \mathrm{mM} \mathrm{FeSO}_{4}=1\right.$ FRAP value) ${ }^{[14]}$.

\section{Results}

\subsection{Determination results of total flavonoids and total saponins}

\subsubsection{Total flavonoids determination result}

Figure 2 shows the determination results of total flavonoids in all membrane separation samples, retentates M1, M2, M3 and permeate MT. The content of total flavonoids in the lyophilized powder of MT was the lowest, M1 was the highest, and M3 was the second highest. It shows that membrane separation technology can be effectively applied to the separation of total flavonoids.

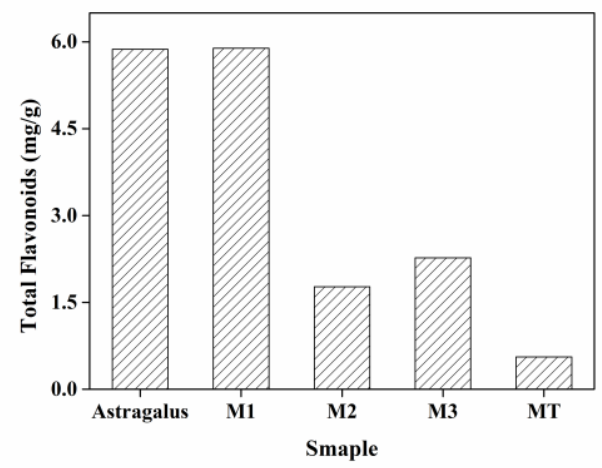

Fig. 2. Contents of total flavonoids in membrane separation samples 


\subsubsection{Total saponins determination result}

The determination results of total saponin in all membrane separation samples are shown in Figure 3. The contents of total astragalus saponins were significantly different, following the order of M3 > M1> M2 > MT. In the freeze-dried powder of M3, saponins content was 2.67 times of that in the feeding astragalus extract. There were almost no astragalus saponins in MT. The results indicate that membrane separation can be used for the separation of total saponins in Astragalus.

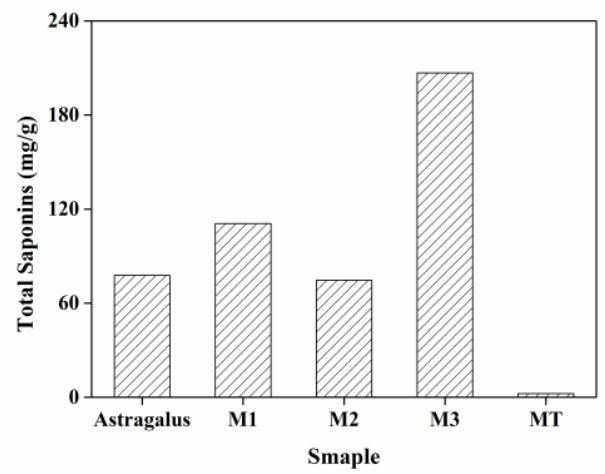

Fig. 3. Contents of total saponins in membrane separation samples

\subsection{Determination results of calycosin-7-O- $\beta$-D- glucoside and Astragaloside IV}

\subsubsection{Calycosin-7-O- $\beta-D-$ glucoside determination result}

Contents of calycosin-7-O- $\beta$-D-glucoside in all membrane separation samples are shown in Figure 4 and the results are similar to those of total flavonoids. Compared with feeding astragalus extract, contents of calycosin-7-O- $\beta$-D-glucoside increased in M1 and M3. MT rarely contained any calycosin-7-O- $\beta$-D-glucoside Results suggest that membrane separation is effective in calycosin-7-O- $\beta$-D-glucoside separation.

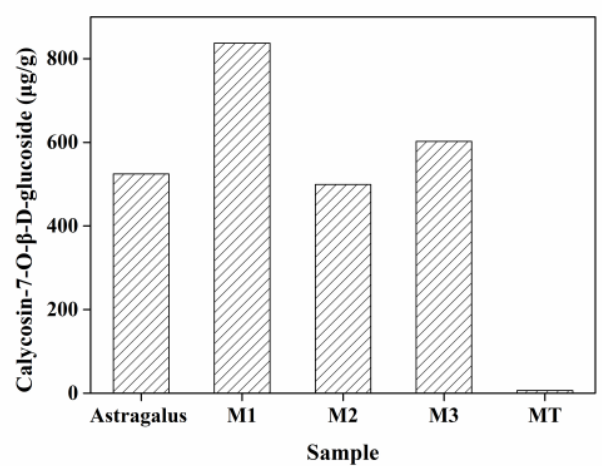

Fig. 4. Contents of calycosin-7-O- $\beta$-D-glucoside in membrane separation samples

\subsubsection{Astragaloside IV determination result}

Contents of Astragaloside IV in M1, M2 and M3 were higher than in the feeding astragalus extract and there was no Astragaloside IV detected in MT (Figure 5). Membrane separation, therefore, can a good choice in separation of Astragaloside IV.

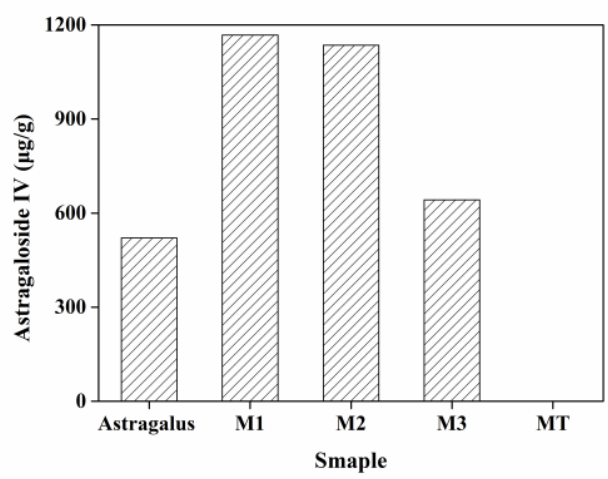

Fig. 5. Contents of Astragaloside IV in membrane separation samples

\subsection{Antioxidant capacities}

\subsubsection{DPPH free radical scavenging activity}

Figure 6 demonstrates the activities of DPPH free radical scavenging of all membrane separation samples. According to the results, the order of the scavenging activities of DPPH free radicals were as follows: MT > M3 $>$ M2 $>$ M1 $>$ feeding astragalus extract. This order is assumed to be the same as the order of total phenolic acids contents of the samples. Phenolic acids contain a large number of phenolic hydroxyl groups which are excellent hydrogen or neutron donors and have been proven to be able to scavenge various free radicals in vivo. Results show that the phenolic acids in astragalus are effective in scavenging DPPH free radical ${ }^{[15]}$.

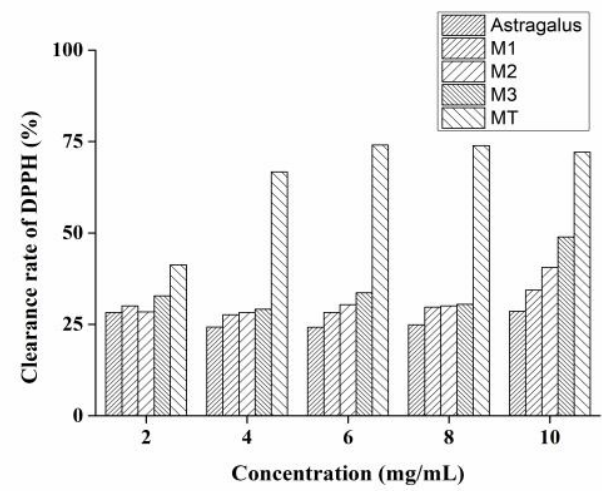

Fig. 6. DPPH free radical scavenging activities of membrane separation samples at different concentrations

\subsubsection{Hydroxyl radical scavenging activity}

The antioxidant capacity of the drug is mainly manifested in its ability to reduce the generation of free radicals and to remove free radicals. As shown in Figure 7, the activity of hydroxyl radical scavenging of astragalus is negatively correlated to its concentration when over $2 \mathrm{mg} / \mathrm{mL}$. This might be ascribed to the 
impact on absorbance in UV/vis detection or the promotion of hydroxyl radical generation by concentrated astragalus extract. Astragalus demonstrated a greater scavenging ability of hydroxyl free radicals than that of VC.

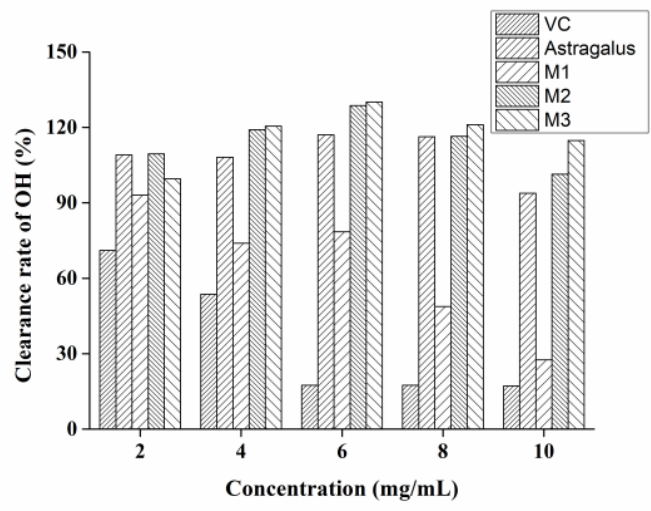

Fig. 7. Hydroxyl free radical scavenging activities of membrane separation samples at different concentrations

\subsubsection{Total antioxidant capacities}

Standard curved of FRAP assay is shown as in Equation 3 and its $\mathrm{R}^{2}$ is 0.992 .

$$
A=-0.0248 C+0.664
$$

$C$ is the concentration of standard $\mathrm{FeSO}_{4}$ solutions and $A$ represents absorbance at $593 \mathrm{~nm}$. Theoretically, FRAP value is positively correlated to the antioxidant capacity of drugs. In Figure 8, antioxidant capacities of each membrane separation sample increase as concentration increases, suggesting a dosage-effect relationship. Among all samples, M1 and MT showed higher FRAP values than the rest and M1 was the strongest. Substances in M1 are greater than $10 \mathrm{kDa}$ in molecular weight, which mainly include polysaccharides and saponins from astragalus, and have strong antioxidant capacities. MT mainly contains chemicals with molecular weight less than $600 \mathrm{Da}$, which are phenolic acids.

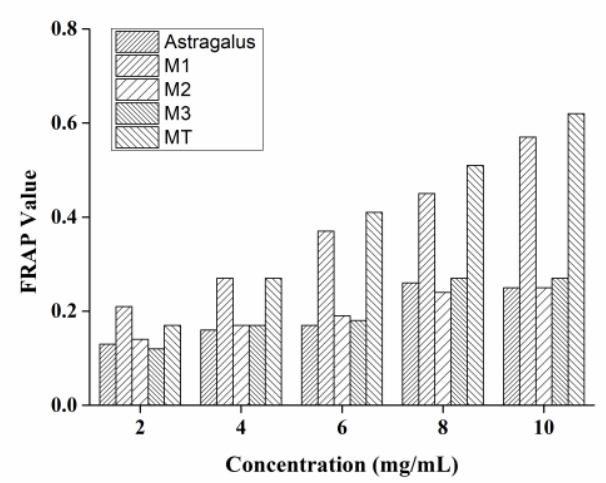

Fig. 8. FRAP values of each membrane separation samples at different concentrations

\section{Discussion}

Conventional approach for extracting efficacious components from astragalus generally employ water extraction and alcohol precipitation method, requiring large amounts of organic reagents, and the processes are cumbersome and time-consuming. In this work, we used ultrafiltration membranes with molecular weight cut-off of $600 \mathrm{Da}, 2.5 \mathrm{kDa}$ and $10 \mathrm{kDa}$. Previous studies have shown that ultrafiltration membranes have high separation efficiencies for the main efficacious components in traditional Chinese medicine, and are short in time and environment-friendly ${ }^{[16-17]}$. Combining the data obtained from UV-vis and HPLC characterizations in this experiment, it can be seen that the results of this experiment are basically consistent with the literature.

Through determination of antioxidant capacities of different membrane separation samples using DPPH assay, hydroxyl free radicals scavenging assay and FRAP assay, we have confirmed that substances from astragalus in different molecular weight ranges differ in antioxidant capacities.

DPPH antioxidant assay demonstrated MT possessed the strongest scavenging activity, and the activity order of the rest samples were as follows: M3 $>$ M2 $>$ M1. Li et.al used ethanol fractionation to extract astragalus polysaccharides and found that the astragalus polysaccharides with relatively small molecular weights have stronger antioxidant capacity ${ }^{[18]}$. Results in this work are in accordance with their finding.

In the hydroxyl radical scavenging experiment, the scavenging rates of M2 and M3 on hydroxyl free radicals were significantly higher than that of other membrane separation samples, and the scavenging activity of each membrane separation sample on hydroxyl free radicals showed a decreasing trend with the increase of concentration. Liu et.al found that the scavenging ability of astragalus for hydroxyl free radicals was higher at a concentration of $0.1 \mathrm{mg} / \mathrm{mL}$, which indicates that the scavenging ability of astragalus for hydroxyl free radicals decreased with the increase of the concentration after $2 \mathrm{mg} / \mathrm{mL}^{[19]}$.

The FRAP assay aimed to compare the redox potentials of membrane separation samples from astragalus. Results show that MT and M1 had stronger redox potentials, which is consistent with the free radical scavenging activities determined by DPPH assay, implying that MT is the strongest antioxidant sample in all astragalus membrane separation samples. Part of the MT is mainly substances with a molecular weight less than $600 \mathrm{Da}$, and the specific types of components still need further qualitative analysis. M1 contains the highest amounts of total saponins, total flavonoids and total polysaccharides, indicating that these types of substance have a certain degree of redox potential.

The application of membrane separation technology to the separation and purification of astragalus aqueous extract has obtained preliminary outcomes. However, we need to optimize the experimental process and prove the advantages of using membrane separation technology in 
natural medicine extraction and purification in a wider range of aspects. In this experiment, only in vitro antioxidation experiments have been applied to show the certain differences in the antioxidant capacities between the different membrane separation cut-off sections of astragalus water extract. Researchers have found through experiments that Astragaloside IV had a certain enhancement on hydrogen peroxide-induced oxidative stress and speculated that Astragaloside IV had the effect of improving the antioxidant capacity of mitochondria [20]. To obtain the specific antioxidant capacities of ultrafiltration membrane separation samples from the water extract of astragalus, pharmacological experiments can be adopted, such as superoxide dismutase (SOD), glutathione peroxidase (GSH-Px), malondialdehyde (MDA) assay, etc. ${ }^{[19]}$.

\section{Acknowledgments}

This work was financially supported by 'Astragalus Collaborative Innovation Center Project of Shanxi Province' (Grant No. HQXTCXZX2016-019).

\section{References}

1. H. Yuan, S.F. Zhang, S.H. Jia, Food Sci. 35, 330334 (2014).

2. D. Turck, J Castenmiller, S.De. Henauw, K.I. Hirsch-Ernst, J. Kearney, A. Maciuk, EFSA J. 18, 5, e06099 (2020).

3. China Pharmacopoeia Commission, Pharmacopoeia of the People's Republic of China. China Medical Science and Technology Press (2015).

4. C.Y. Wang, W.M. Li, Y. Gao, J. Liaoning Uni. of Traditional Chinese Med. 19, 44-47 (2017).

5. S.M. Qin, J.Y. Lin, K. Huang, Chinese Arch. of Traditional Chinese Med. 35, 699-701 (2017).

6. Q, Zhang, W.Y. Gao, S.L. Man, China J. of Chinese Materia Medica. 37, 3203-3207 (2012).

7. H.G, Chen, X. Zhou, J. Zhang. Carbohydrate Polymers, 111, 567-575(2014).

8. L.W. Guo, W.H. Xing, H.X. Zhu, Chinese Traditional and Herbal Drugs. 48, 16, 3267-3279 (2017).

9. C. Yiengveerachon, S. Yoshikawa, H. Matsumoto, S. Ookawara. J. of Food Process Engi, 43, 4 (2020).

10. S. Chakraborty, R. Uppaluri, C. Das, Innova Food Sci \& Emerging Tech. 102382 (2020).

11. C. M. Roberto, G. Boczkaj, E. Gontarek, A. Cassano, V. Fila, Trends in Food Sci \& Tech, 95, 219-232. (2020).

12. Z.R. Wang, Uni of zhengzhou. (2010).

13. Y. Wu, H. Yang, T.S. Huang, Chinese J. of Exper. Traditional Med. Formulae. 18,193-197 (2014).

14. L. Yang, Guangdong Pharmaceutical University (2017).

15. N. Shi, Hunan Normal University (2011).
16. Y. Yang, W.Z. Wang, B.H. He, Chem \& Bioengi. 27, 5-7 (2010).

17. L.L. Zou, L. J. Wang, Y.F. Wang, et al., Feed Res. 6, 22-25 (2017).

18. H.F. Li, S. B. Guo, S. L. Man, Y.Y. Fan, T.T. Wang, X. Li, W.Y. Gao, China J. of Chinese Materia Medica.40, 2112-2116 (2015).

19. L. Liu, K. Li, X. Hao, G.Z. Wang, X.M. Qin, G.H. Du, X. Zhang, Chem. J. of Chinese Uni. 37, 21682175 (2016).

20. Y.Q. Chen, D.Y. Sun, H.F. Wu, Z.P. Bian, X.J. Chen, D. Yang, JNMU Natural Sci. 37, 949-954 (2017). 